TOPOLOGICAL ALGEBRAS, THEIR APPLICATIONS,

AND RELATED TOPICS

BANACH CENTER PUBLICATIONS, VOLUME 67

INSTITUTE OF MATHEMATICS

POLISH ACADEMY OF SCIENCES

WARSZAWA 2005

\title{
BOUNDED POINT EVALUATIONS FOR MULTICYCLIC OPERATORS
}

\author{
M. EL GUENDAFI \\ Université Lille 1 \\ UFR de Mathématiques, UMR-CNRS 8524, Bât. M2 \\ F-59655 Villeneuve Cedex, France \\ E-mail: mohamed.elguendafi@math.univ-lille1.fr \\ M. MBEKHTA \\ Université Lille 1 \\ UFR de Mathématiques, UMR-CNRS 8524, Bât. M2 \\ F-59655 Villeneuve Cedex, France \\ E-mail: mostafa.mbekhta@math.univ-lille1.fr \\ E. H. ZEROUALI \\ Département de Mathématiques et Informatique \\ Faculté des Sciences de Rabat \\ BP 1014 Rabat, Maroc \\ E-mail: zerouali@fsr.ac.ma
}

\begin{abstract}
Let $T$ be a multicyclic operator defined on some Banach space. Bounded point evaluations and analytic bounded point evaluations for $T$ are defined to generalize the cyclic case. We extend some known results on cyclic operators to the more general setting of multicyclic operators on Banach spaces. In particular we show that if $T$ satisfies Bishop's property $(\beta)$, then

$$
\mathcal{B}_{a}(T)=\mathcal{B}(T) \backslash \sigma_{a p}(T)
$$

We introduce the concept of analytic structures and we link it to different spectral quantities. We apply this concept to retrieve in an easy way a theorem of D. Herrero and L. Rodman: the set of cyclic $n$-tuples for a multicyclic operator $T$ is dense if and only if $\mathcal{B}_{a}(T)=\emptyset$.
\end{abstract}

2000 Mathematics Subject Classification: Primary 47B; Secondary 47A.

Key words and phrases: multicyclic operators, bounded point evaluations, singular spectrum, Bishop's property $(\beta)$.

The paper is in final form and no version of it will be published elsewhere. 
1. Introduction. Throughout this paper, $\mathcal{X}$ will denote an infinite dimensional Banach space and $\mathcal{L}(\mathcal{X})$ the algebra of all bounded operators on $\mathcal{X}$. For $T \in \mathcal{L}(\mathcal{X})$, let $N(T), R(T)$, $\sigma(T), \sigma_{p}(T)$ and $\sigma_{a p}(T)$ denote the kernel, the range, the spectrum, the point spectrum (that is, the set of eigenvalues) and the approximate point spectrum respectively. For a subset $E$ of $\mathcal{X}$, we let $\operatorname{span}\{E\}$ be the closure of the linear space generated by $E$. If $F$ is any subset of of the complexe plane, write $\operatorname{int}(F)$ for the interior points of $F$ and $\operatorname{cl}(F)$ for the closure of $F$.

1.1. Bounded point evaluations for $P^{t}(\mu)$. Bounded point evaluations were first defined in a natural way for some Banach function spaces. To be precise, consider a compactly supported nonnegative measure $\mu$ and let $P^{t}(\mu)$ be the closure in $L^{t}(\mu)$ of all complex polynomials $(1 \leq t<\infty)$.

A complex number $\lambda$ is a bounded point evaluation for $P^{t}(\mu)$ if there exists a constant $M>0$ such that

$$
|P(\lambda)| \leq M\|P\|_{L^{t}(\mu)},
$$

for any polynomial $P$. The set of all bounded point evaluations for $P^{t}(\mu)$ will be denoted by $\mathcal{B}\left(P^{t}(\mu)\right)$. It is clear that if $\lambda \in \mathcal{B}\left(P^{t}(\mu)\right)$, then the mapping $P \mapsto P(\lambda)$ extends to a bounded linear functional on $P^{t}(\mu)$. By the Riesz representation theorem and the Hahn-Banach theorem, there exists $k_{\lambda} \in L^{s}(\mu)\left(\frac{1}{t}+\frac{1}{s}=1\right)$ such that

$$
P(\lambda)=\int P(z) k_{\lambda}(z) d \mu(z) \quad \text { for every polynomial } P .
$$

Note in passing that $k_{\lambda}$ need not be unique. However if, $\lambda \in \mathcal{B}\left(P^{t}(\mu)\right)$ an evaluation map is defined by

$$
\lambda \rightarrow \hat{f}(\lambda)=\int f(z) k_{\lambda}(z) d \mu(z),
$$

for every $f \in P^{t}(\mu)$ and does not depend of the choice of $k_{\lambda}$. It is known that $f=\hat{f}$ $\mu$-almost everywhere on $\mathcal{B}\left(P^{t}(\mu)\right)$.

We will say that $\lambda_{0} \in \operatorname{int}\left(\mathcal{B}\left(P^{t}(\mu)\right)\right)$ is an analytic bounded point evaluation for $P^{t}(\mu)$ if the mapping $\lambda \rightarrow \hat{f}(\lambda)$ is analytic at $\lambda_{0}$ for arbitrary $f \in P^{t}(\mu)$. We denote by $\mathcal{B}_{a}\left(P^{t}(\mu)\right)$ the set of all bounded point evaluations for $P^{t}(\mu)$.

The sets $\mathcal{B}\left(P^{t}(\mu)\right)$ and $\mathcal{B}_{a}\left(P^{t}(\mu)\right)$ are widely studied and have a lot of applications. For brevity, we mention only one example. To show that subnormal operators are reflexive, J. E. McCarthy [15, Theorem 1] used in a crucial way the existence of bounded point evaluations for pure subnormal operators.

REMARK 1. The following properties of $\mathcal{B}_{a}\left(P^{t}(\mu)\right)$ are easy to establish.

1) A complex number $\lambda_{0}$ is an analytic bounded point evaluation of $P^{t}(\mu)$ if and only if there exist $M>0$ and $r>0$ such that $|P(z)| \leq M\|P\|_{L^{t}(\mu)}$ for every $P$ and every $z$ such that $\left|\lambda_{0}-z\right|<r$.

2) Clearly $\mathcal{B}_{a}\left(P^{t}(\mu)\right)$ is contained in the interior of $\mathcal{B}\left(P^{t}(\mu)\right)$. In fact by using the Baire category theorem, one can show that

$$
\mathcal{B}_{a}\left(P^{t}(\mu)\right) \subset \operatorname{int}\left(\mathcal{B}\left(P^{t}(\mu)\right)\right) \subset \operatorname{cl}\left(\mathcal{B}\left(P^{t}(\mu)\right)\right) .
$$

See $[28,33]$ for example. 
3) By using the maximum modulus principle for analytic functions, we obtain that connected components of $\mathcal{B}_{a}\left(P^{t}(\mu)\right)$ are simply connected.

4) It is obvious that $\mathcal{B}_{a}\left(P^{t}(\mu)\right)$ and $\mathcal{B}\left(P^{t}(\mu)\right)$ vary with $\mu$. In [1], measures such that these sets vary also with $t$ are given.

We recall below some results from the literature that have motivated our work. We refer to original papers for complete proofs.

Our starting result is a theorem due to T. Trent in the case $t=2$. An adaptation of Trent's proof will permit us to obtain the same result for $P^{t}(\mu)(1 \leq t<+\infty)$.

ThEOREM 1 ([28, Theorem 1.1]). Let $\mu$ be a nonnegative compactly supported measure and $1 \leq t<+\infty$. Then

$$
\mathcal{B}_{a}\left(P^{t}(\mu)\right)=\sigma\left(M_{z}^{t}\right) \backslash \sigma_{a p}\left(M_{z}^{t}\right)=\mathcal{B}\left(P^{t}(\mu)\right) \backslash \sigma_{a p}\left(M_{z}^{t}\right) .
$$

Here $M_{z}^{t}$ is the multiplication operator in $P^{t}(\mu)$ by the independent variable $z$.

It is not hard to see that if $\mathcal{B}_{a}\left(P^{t}(\mu)\right) \neq \emptyset$, then $P^{t}(\mu) \neq L^{t}(\mu)$. To obtain the reverse implication is much more difficult. This has interested various authors. In particular this will allow a description of the approximation by polynomials property in terms of analytic bounded point evaluations. A complete description was given in [27]. That is, $P^{t}(\mu)=$ $L^{t}(\mu)$ if and only if $\mathcal{B}_{a}\left(P^{t}(\mu)\right)=\emptyset$. However, the problem remains of the description of measures such that $\mathcal{B}_{a}\left(P^{t}(\mu)\right)=\emptyset$. Particular cases are given in [16, 28]. Some sufficient conditions can be found in [27] (see also [30] and [6, Chap. 8, Theorem 4.2]).

The space $P^{t}(\mu)$ is said to be pure if it contains no $L^{t}$ space as a direct summand.

Theorem $2\left(\left[27\right.\right.$, Theorem 4.11]). If $P^{t}(\mu)$ is pure then the closure of $\mathcal{B}_{a}\left(P^{t}(\mu)\right)$ contains the support of $\mu$.

Consequently, if the support of $\mu$ has nonempty interior, and $P^{t}(\mu)$ is pure, then analytic bounded point evaluations exist.

1.2. Bounded point evaluations for cyclic operators. L. R. Williams extended the concept of bounded point evaluations and analytic bounded point evaluations to the class of all cyclic operators. See [33]. We present in this section this extension. Even if it is not difficult to adapt William's results to the more general setting of Banach spaces, we restrict ourselves to the Hilbert space case.

Let $T$ be a bounded operator defined on some Hilbert space $H$. The operator $T$ is said to be cyclic if there exists $y \in H$ such that $\operatorname{span}\left\{T^{n} y ; n \geq 0\right\}=H$. Such an element is then called a cyclic vector for $T$. A point $\lambda_{0} \in \sigma(T)$ is a bounded point evaluation for $T$ if there exists $M>0$ such that

$$
\left|P\left(\lambda_{0}\right)\right| \leq M\|P(T) y\|
$$

for every polynomial $P$. The set of all bounded point evaluations for $T$ is denoted similarly by $\mathcal{B}(T)$. For $\lambda_{0} \in \mathcal{B}(T)$, the evaluation map

$$
w_{\lambda_{0}}: P(T) y \mapsto P\left(\lambda_{0}\right)
$$

can be extended by density on $H$ to some bounded linear functional. By the Riesz representation theorem, there exists a unique vector $k\left(\lambda_{0}\right)$ such that for any polynomial $P$, 
we have

$$
w_{\lambda_{0}}(P(T) y)=\left\langle P(T) y, k\left(\lambda_{0}\right)\right\rangle=P\left(\lambda_{0}\right) .
$$

In particular, $k\left(\lambda_{0}\right) \in N\left(T-\lambda_{0}\right)^{*}$ and $\left\langle y, k\left(\lambda_{0}\right)\right\rangle=1$.

Conversely, take $k\left(\lambda_{0}\right) \in N\left(T-\lambda_{0}\right)^{*}$. Since $y$ is a cyclic vector for $T$, it follows that $\left\langle y, k\left(\lambda_{0}\right)\right\rangle \neq 0$. Without loss of generality, we can suppose that $\left\langle y, k\left(\lambda_{0}\right)\right\rangle=1$; then by Cauchy's inequality, for any polynomial $P$ we get

$$
\left|P\left(\lambda_{0}\right)\right|=|\langle P(T) y, k\rangle| \leq\left\|k\left(\lambda_{0}\right)\right\|\|P(T) y\| .
$$

Thus $\lambda_{0} \in \mathcal{B}(T)$ and consequently

$$
\mathcal{B}(T)=\overline{\sigma_{p}\left(T^{*}\right)}=\left\{\lambda \in \mathbb{C} ; \operatorname{dim}\left(N(T-\lambda)^{*}\right)=1\right\} .
$$

Observe that equation (8) shows in particular that the set of bounded point evaluations does not depend on the choice of the cyclic vector.

We say that $\lambda_{0} \in \operatorname{int}(\mathcal{B}(T))$ is an analytic bounded point evaluation for $T$ if the evaluation map $\lambda \mapsto \widehat{x}(\lambda)=\langle x, k(\lambda)\rangle$ is analytic at $\lambda_{0}$, for every $x \in H$. We denote by $\mathcal{B}_{a}(T)$ the set of all such points.

The proposition below provides a characterization of $\mathcal{B}_{a}(T)$ generalizing the case of subnormal operators. The proof is similar and is omitted (see [6]).

Proposition 1. Let $T$ be a cyclic operator and $\mathcal{O}$ be an open subset of $\mathcal{B}(T)$. The following properties are equivalent:

1. $\mathcal{O} \subset \mathcal{B}_{a}(T)$.

2. The mapping $\lambda \mapsto k(\lambda) \in H$ is continuous on $\mathcal{O}$.

3. The mapping $\lambda \mapsto\|k(\lambda)\|$ is bounded on compact sets of $\mathcal{O}$.

REMARK 2 . Let $M_{z}$ be the multiplication operator in $P^{t}(\mu)$ by the independent variable $z$. Then, clearly

$$
\mathcal{B}\left(M_{z}\right)=\mathcal{B}\left(P^{t}(\mu)\right) \text { and } \mathcal{B}_{a}\left(M_{z}\right)=\mathcal{B}_{a}\left(P^{t}(\mu)\right) .
$$

From the remark above the following general question arises naturally. Which results known for bounded point evaluations for Banach function spaces can be carried over to arbitrary cyclic operators? Some of these results does not require any specific properties of functions like Equation (4) and hence are trivially true for all cyclic operators. In general it is not automatic to extend the other results. The following question is stated in [33]. Does the equality

$$
\mathcal{B}_{a}(T)=\sigma(T) \backslash \sigma_{a p}(T)
$$

hold for all cyclic operators?

In the same paper the inclusion $\sigma(T) \backslash \sigma_{a p}(T) \subseteq \mathcal{B}_{a}(T)$ is proved for arbitrary cyclic operators, [33, Proposition 1.3], see also [21, Proposition 2.2]. The other inclusion fails to be true in general, see $[5,21]$, where counterexamples are given. It is also known from [21] that equality (5) is valid for a large class of operators containing cyclic hyponormal operators.

TheOREM 3 ([21, Théorème 3.1]). Let T be a cyclic operator with Bishop's property $(\beta)$. Then

$$
\mathcal{B}_{a}(T)=\sigma(T) \backslash \sigma_{a p}(T)
$$


For the definition of operators with $(\beta)$, see section 5 .

The preceding result has been extended to the case of rationally cyclic operators on Banach spaces with Bishop's property $(\beta)$ in [25].

In this paper we define bounded point evaluations for multicyclic operators on infinite dimensional Banach spaces. We extend many results from [21] to this situation.

The paper is organized as follows.

In section 2 we define bounded point evaluations and analytic bounded point evaluations associated with a multicyclic operator. We provide some results for the cyclic case. In particular we show that

$$
\mathcal{B}(T)=\left\{\lambda \in \sigma(T): \operatorname{dim}\left(N(T-\lambda)^{*}\right)=n\right\} .
$$

Analytic structures associated with multicyclic operators are introduced in section 3. We also discuss the link between analytic bounded point evaluations, the analytic structure in the sense of D. A. Herrero in [13] and the analytic structures defined here. We also characterize analytic structures in terms of the analytic core.

In section 4 we link the analytic structures to the singular spectrum. It is shown in particular that, if $T$ is an $n$-multicyclic operator and $m \leq n$, then

$$
\mathcal{B}^{m}(T) \backslash \sigma_{s}(T)=\mathcal{B}_{a}^{m}(T) \backslash \sigma_{s-F}(T),
$$

where $\mathcal{B}^{m}(T)=\left\{\lambda \in \mathbb{C} ; \quad \operatorname{dim}\left(N(T-\lambda)^{*}\right)=m\right\}$, and where $\sigma_{s}(T), \sigma_{s-F}(T)$ and $\mathcal{B}_{a}^{m}(T)$ stand for the singular spectrum, the semi-Fredholm spectrum and the analytic structure of dimension $m$ respectively. We also show that connected components of $\mathcal{B}_{a}(T)$ remain simply connected in the case of multicyclic operators.

The role of Bishop's property $(\beta)$ in the description of $\mathcal{B}_{a}(T)$ is investigated in section 5 . The main result of this section is

$$
\mathcal{B}_{a}(T)=\mathcal{B}(T) \backslash \sigma_{a p}(T),
$$

where $T$ is a multicyclic operator with property $(\beta)$.

In section 6 , we apply the concept of analytic bounded point evaluations to retrieve in an easy way the following theorem of D. A. Herrero and L. Rodman: The set of all cyclic $n$-tuples for $T$ is dense in $\mathcal{X}^{(n)}$ if and only if $\mathcal{B}_{a}(T)=\emptyset$. We then derive some known results on operators with $n$-multicyclic powers.

2. Bounded point evaluations for multicyclic operators. In what follows $\langle$,$\rangle de-$ notes the natural duality between $\mathcal{X}$ and its topological dual $\mathcal{X}^{*}$. For $T \in \mathcal{L}(\mathcal{X})$, we denote by $T^{*} \in \mathcal{L}\left(\mathcal{X}^{*}\right)$ its adjoint operator $T$. As usual, we will write $(T-\lambda)^{*}=T^{*}-\lambda$ in the Banach space case and $(T-\lambda)^{*}=T^{*}-\bar{\lambda}$ in the Hilbert space setting.

Let $T$ be a bounded operator on some Banach space $\mathcal{X}$ and $n \geq 1$ be an integer. We say that $T$ is a multicyclic operator of order $n$ ( $n$-multicyclic for short) if there exist $n$ vectors $y_{1}, y_{2}, \ldots, y_{n} \in \mathcal{X}$ such that $\mathcal{X}=\operatorname{span}\left\{T^{m} y_{i} ; i=1,2, \ldots, n, m \geq 0\right\}$ and if for any $n-1$ vectors $x_{1}, x_{2}, \ldots, x_{n-1}$ in $\mathcal{X}$, the subspace $\operatorname{span}\left\{T^{m} x_{i}, m \geq 0,1 \leq i \leq n\right\}$ is proper. The $n$-tuple $\left(y_{1}, y_{2}, \ldots, y_{n}\right)$ is then called a cyclic $n$-tuple for $T$.

We shall denote by $C_{n}(\mathcal{X})$ the set of all $n$-multicyclic operators on $\mathcal{X}$, and for $T \in$ $C_{n}(\mathcal{X})$, the set of all cyclic $n$-tuples for $T$ is denoted $C_{n}(T)$. 
Bounded point evaluations for a multicyclic operator $T$ on a Banach space $\mathcal{X}$ are defined in the following way: Let $\left(y_{1}, \ldots, y_{n}\right) \in C_{n}(T)$. A complex number $\lambda_{0} \in \sigma(T)$ is said to be a bounded point evaluation for $T$ if there exists $M>0$ such that

$$
\sum_{i=1}^{n}\left|P_{i}\left(\lambda_{0}\right)\right| \leq M\left\|\sum_{i=1}^{n} P_{i}(T) y_{i}\right\|
$$

for every family of complex polynomials $\left\{P_{1}, P_{2}, \ldots, P_{n}\right\}$. The set of all bounded point evaluations for $T$ will be denoted by $\mathcal{B}(T)$.

For $\lambda_{0} \in \mathcal{B}(T)$ and for $j \in\{1, \ldots, n\}$, we have

$$
\left|P_{j}\left(\lambda_{0}\right)\right| \leq M\left\|\sum_{i=1}^{n} P_{i}(T) y_{i}\right\|
$$

This implies that the linear mapping

$$
w^{j}\left(\lambda_{0}\right):\left\{\sum_{i=1}^{n} P_{i}(T) y_{i} ; P_{1}, P_{2}, \ldots, P_{n} \in \mathcal{P}\right\} \rightarrow \mathbb{C}, \quad \sum_{i=1}^{n} P_{i}(T) y_{i} \mapsto P_{j}\left(\lambda_{0}\right)
$$

is well defined (here $\mathcal{P}$ is the set of all complex polynomials). Now by $n$-multicyclicity the set $\left.\left\{\sum_{i=1}^{n} P_{i}(T) y_{i} ; P_{1}, P_{2}, \ldots, P_{n} \in \mathcal{P}\right\}\right)$ is a dense subset of $\mathcal{X}$. The mapping $w^{j}\left(\lambda_{0}\right)$ can be extended to a bounded linear functional defined on $\mathcal{X}$. Denote by $k_{j}\left(\lambda_{0}\right) \in \mathcal{X}^{*}$ such an extension. Then we have

$$
w^{j}\left(\lambda_{0}\right)\left(\sum_{i=1}^{n} P_{i}(T) y_{i}\right)=\left\langle\sum_{i=1}^{n} P_{i}(T) y_{i}, k_{j}\left(\lambda_{0}\right)\right\rangle=P_{j}\left(\lambda_{0}\right), \quad \forall\left\{P_{1}, P_{2}, \ldots, P_{n}\right\} \in \mathcal{P} .
$$

In particular,

$$
k_{j}\left(\lambda_{0}\right) \in N\left(T^{*}-\lambda_{0}\right) \text { and }\left\langle y_{i}, k_{j}\left(\lambda_{0}\right)\right\rangle=\delta_{i j} .
$$

Conversely, if $k_{1}\left(\lambda_{0}\right), k_{2}\left(\lambda_{0}\right), \ldots, k_{n}\left(\lambda_{0}\right) \in N\left(T^{*}-\lambda_{0}\right)$ satisfy Equation (11) then, applying Cauchy's inequality, for arbitrary polynomials $\left\{P_{1}, P_{2}, \ldots, P_{n}\right\} \in \mathcal{P}$ and $j \in$ $\{1,2, \ldots, n\}$, yields

$$
\sum_{j=1}^{n}\left|P_{j}\left(\lambda_{0}\right)\right|=\sum_{j=1}^{n}\left|\mathcal{B}(T)\left\langle\sum_{i=1}^{n} P_{i}(T) y_{i}, k_{j}\right\rangle\right| \leq\left(n \sup _{j}\left\|k_{j}\right\|\right)\left\|\sum_{i=1}^{n} P_{i}(T) y_{i}\right\| .
$$

Finally $\lambda_{0} \in \mathcal{B}(T)$.

The following proposition will be useful in the sequel.

Proposition 2. Let $T \in \mathcal{C}_{n}(\mathcal{X})$ be $n$-multicyclic and $\left(y_{1}, y_{2}, \ldots, y_{n}\right) \in \mathcal{C}_{n}(T)$ be an associated cyclic $n$-tuple. Then for every $\lambda \in \mathbb{C}$ we have

(i) $\operatorname{cl}(\mathcal{R}(T-\lambda))+\operatorname{span}\left\{y_{1}, y_{2}, \ldots, y_{n}\right\}=\mathcal{X}$,

(ii) $\operatorname{dim} N(T-\lambda)^{*} \leq n$.

Proof. ( $i)$ Let $\lambda$ be a complex number. Since $\left(y_{1}, y_{2}, \ldots, y_{n}\right) \in \mathcal{C}_{n}(T)$, we have

$$
\begin{aligned}
c l(\mathcal{R}(T-\lambda))+\operatorname{span}\left\{y_{1}, y_{2}, \ldots, y_{n}\right\}= & c l((T-\lambda)(\mathcal{X}))+\operatorname{span}\left\{y_{1}, y_{2}, \ldots, y_{n}\right\} \\
\supseteq & \operatorname{span}\left\{(T-\lambda)^{k} y_{i} ; k \geq 1,1 \leq i \leq n\right\} \\
& +\operatorname{span}\left\{y_{1}, y_{2}, \ldots, y_{n}\right\} \\
\supseteq & \operatorname{span}\left\{T^{k} y_{i} ; k \geq 0,1 \leq i \leq n\right\}=\mathcal{X} .
\end{aligned}
$$


The last inclusion follows from the fact that $T^{k} y_{i}=\left(T^{k}-\lambda^{k}\right) y_{i}+\lambda^{k} y_{i} \in \operatorname{span}\{R(T-$ $\left.\lambda), y_{i}\right\}$.

$($ ii $)$ is a direct corollary of $(i)$.

In the case of cyclic operators Equality (8) provides an algebraic characterization of $\mathcal{B}(T)$. For $n$-multicyclic operators Equality (8) fails to be true if $n \geq 2$, as shown by the following example:

ExAmPle 1. Let $H$ be an infinite dimensional separable Hilbert space and $\left(e_{n}\right)_{n \in \mathbb{N}}$ be an orthonormal basis of $H$. Consider the linear bounded operator defined on the basis by:

$$
S e_{n}= \begin{cases}e_{n+1} & \text { if } n \geq 1, \\ e_{2} & \text { if } n=0,\end{cases}
$$

The adjoint $S^{*}$ of $S$ is given by

$$
S^{*} e_{n}= \begin{cases}e_{n-1} & \text { if } n \geq 3 \\ e_{0}+e_{1} & \text { if } n=2 \\ 0 & \text { if } n=0,1\end{cases}
$$

Then

1. $S$ is 2 -multicyclic. Indeed, $\operatorname{dim} N\left(S^{*}\right)=2$, and $H=\operatorname{span}\left\{e_{0}, S^{n} e_{1} ; n \geq 0\right\}$. Hence $S \in \mathcal{C}_{2}(H)$.

2. $D(0,1) \subset \sigma_{p}\left(S^{*}\right)$. In fact if, $\lambda \in D(0,1)$, then it is clear that $(S-\lambda)^{*}\left(\bar{\lambda} e_{0}+\right.$ $\left.\sum_{n>1} \bar{\lambda}^{n} e_{n}\right)=0$.

3. $\mathcal{B}(S) \subset\{0\}$. To see this, take $\lambda \in \mathcal{B}(S)$; then for any polynomials $P$ and $Q$, we will have

$$
|P(\lambda)|+|Q(\lambda)| \leq\left\|P(S) e_{0}+Q(S) e_{1}\right\| .
$$

In particular, if $P=-Q=X$, we obtain $2|\lambda| \leq\left\|S e_{0}-S e_{1}\right\|=0$. Hence $\mathcal{B}(S) \subset$ $\{0\} \neq \sigma_{p}\left(S^{*}\right)$.

The algebraic characterization of $\mathcal{B}(T)$ in the case of multicyclic operators is given by

TheOREM 4. Let $T$ be an $n$-multicyclic operator defined on a Banach space $\mathcal{X}$. Then

$$
\mathcal{B}(T)=\left\{\lambda \in \mathbb{C} ; \operatorname{dim} N\left(T^{*}-\lambda\right)=n\right\} .
$$

We will need the following lemma. The proof is a straightforward computation.

Lemma 1. Let $T$ be an n-multicyclic operator on $\mathcal{X},\left(y_{1}, y_{2}, \ldots, y_{n}\right) \in \mathcal{C}_{n}(T)$ and $k_{1}, k_{2}, \ldots, k_{n} \in N\left(T^{*}-\lambda\right)$. The following properties are equivalent:

1. $k_{1}, k_{2}, \ldots, k_{n}$ are linearly independent vectors of $\mathcal{X}$.

2. $\operatorname{det}\left(\left\langle y_{i}, k_{j}\right\rangle_{i j}\right) \neq 0$.

In particular,

$$
\left\{k_{1}, k_{2}, \ldots, k_{n}\right\} \text { is a basis of } N\left(T^{*}-\lambda\right) \Leftrightarrow \operatorname{det}\left(\left\langle y_{i}, k_{j}\right\rangle_{i, j}\right) \neq 0 .
$$

Proof of Theorem 4. The inclusion $\mathcal{B}(T) \subset\left\{\lambda \in \mathbb{C} ; \operatorname{dim} N\left(T^{*}-\lambda\right)=n\right\}$ follows directly from Equation (11). Now, let $\lambda \in \mathbb{C}$ be such that $N\left(T^{*}-\lambda\right)=\operatorname{span}\left\{k_{1}, k_{2}, \ldots, k_{n}\right\}$ is $n$-dimensional. By Lemma 1 , the matrix $\left(\left\langle y_{i}, k_{j}\right\rangle_{i j}\right)$ is invertible. Let $A=\left(a_{i j}\right)_{i, j}$ be its 
inverse and let $l_{j}(\lambda)=\sum_{i=1}^{n} a_{i, j} k_{i}$ with $j=1,2, \ldots, n$. Then we have $\left\{l_{1}, l_{2}, \ldots, l_{n}\right\} \in$ $N(T-\lambda)^{*}$ and $\left\langle y_{j}, l_{i}\right\rangle=\delta_{i j}$. Consequently $\lambda \in \mathcal{B}(T)$.

Let $T \in \mathcal{C}_{n}(\mathcal{X})$ be $n$-multicyclic and let $\left(y_{1}, y_{2}, \ldots, y_{n}\right)$ be a cyclic $n$-tuple for $T$. Then there exists a unique family $\left\{k_{1}, k_{2}, \ldots, k_{n}\right\}$ of $\mathcal{X}$-valued functions defined on $\mathcal{B}(T)$ such that for every $\lambda \in \mathcal{B}(T)$ we have

$$
\operatorname{span}\left\{k_{1}(\lambda), k_{2}(\lambda), \ldots, k_{n}(\lambda\}=N\left(T^{*}-\lambda\right) \text { and }\left\langle y_{i}, k_{j}(\lambda)\right\rangle=\delta_{i j} .\right.
$$

We will also say that $\lambda_{0} \in \operatorname{int}(\mathcal{B}(T))$ is an analytic bounded point evaluation for $T$ if the mapping $\lambda \mapsto \widehat{y}_{i}(\lambda)=\left\langle y, k_{i}(\lambda)\right\rangle$ is analytic at $\lambda_{0}$ for every $y \in \mathcal{X}$, and for $i=1,2, \ldots, n$. The set of all analytic bounded point evaluations for $T$ is denoted by $\mathcal{B}_{a}(T)$.

Adapting the proofs given for cyclic operators (see [21, 33], for example), Proposition 1 is generalized as follows:

Proposition 3. Let $T \in \mathcal{C}_{n}(\mathcal{X})$ and $\mathcal{O}$ be an open subset contained in $\mathcal{B}(T)$. The following are equivalent:

1. $\mathcal{O} \subset \mathcal{B}_{a}(T)$.

2. The mapping $\lambda \mapsto k_{i}(\lambda)$ is continuous on $\mathcal{O}$ for every $1 \leq i \leq n$.

3. The mapping $\lambda \mapsto \sup _{1 \leq i \leq n}\left\|k_{i}(\lambda)\right\|$ is bounded on compact subsets of $\mathcal{O}$.

3. Analytic structures. For $\lambda \in \mathbb{C}$ and $\delta>0$, denote by $D(\lambda, \delta)$ the open disc centered at $\lambda$ and of radius $\delta$ and denote by $\bar{D}(\lambda, \delta)$ the corresponding closed disc.

Let $T$ be an $n$-multicyclic operator and $1 \leq m \leq n$ be an integer. Set

$$
\mathcal{B}^{m}(T)=\left\{\lambda \in \sigma(T): \operatorname{dim} N(T-\lambda)^{*}=m\right\} .
$$

REMARK 3. If $T$ is $n$-multicyclic then

1) $\mathcal{B}(T)=\mathcal{B}^{n}(T)$ (see Proposition 3$)$;

2) $\bigcup_{m=1}^{n} \mathcal{B}^{m}(T)=\sigma_{p}\left(T^{*}\right)$.

We will say that $\lambda_{0} \in \sigma(T)$ is in the analytic structure of dimension $m$ of $T$ (and we will write $\left.\lambda_{0} \in \mathcal{B}_{a}^{m}(T)\right)$ if there exists $\delta>0$ such that $D\left(\lambda_{0}, \delta\right) \subseteq \mathcal{B}^{m}(T)$ and there exist analytic functions $k_{1}, k_{2}, \ldots, k_{m}$ on $D\left(\lambda_{0}, \delta\right)$ satisfying

$$
N\left(T^{*}-z\right)=\operatorname{span}\left\{k_{1}(z), k_{2}(z), \ldots, k_{m}(z)\right\}, \quad \text { for every } z \in D\left(\lambda_{0}, \delta\right) .
$$

It is clear from the definitions that analytic structures do not depend on the choice of the cyclic $n$-tuple of $T$. In the following theorem we show that the $n$-dimensional analytic structure is precisely the set of analytic bounded point evaluations.

TheOREM 5. Let $T$ be an $n$-multicyclic operator on a Banach space $\mathcal{X}$. Then

$$
\mathcal{B}_{a}(T)=\mathcal{B}_{a}^{n}(T)
$$

Proof. The inclusion $\mathcal{B}_{a}(T) \subseteq \mathcal{B}_{a}^{n}(T)$ is clear. Suppose now that there exists an open neighbourhood $\mathcal{O}$ of 0 contained in $\mathcal{B}(T)$ and $n$ analytic functions $\varphi_{1}, \varphi_{2}, \ldots, \varphi_{n}$ on $\mathcal{O}$ such that for every $z \in \mathcal{O}$ we have $N\left(T^{*}-z\right)=\operatorname{span}\left\{\varphi_{1}(z), \varphi_{2}(z), \ldots, \varphi_{n}(z)\right\}$. Let $\left(y_{1}, y_{2}, \ldots, y_{n}\right) \in \mathcal{C}_{n}(T)$ be a cyclic $n$-tuple for $T$. The mapping

$$
H: \mathcal{O} \rightarrow \mathcal{M}_{n, n}(\mathbb{C}), \quad z \mapsto H(z):=\left(\left\langle y_{i}, \varphi_{j}(z)\right\rangle_{i j}\right)
$$


is continuous. Using Lemma 1 we deduce that $H(z)$ is invertible for every $z \in \mathcal{O}$. Let $H^{-1}(z)=\left(a_{i j}(z)\right)_{i j}$ be the inverse matrix of $H(z)$. It is not hard to see that the mapping

$$
K: \mathcal{O} \rightarrow \mathbb{C}^{n}, \quad z \mapsto\left(\sum_{i=1}^{n} a_{i 1}(z) \varphi_{i}(z), \sum_{i=1}^{n} a_{i 2}(z) \varphi_{i}(z), \ldots, \sum_{i=1}^{n} a_{i n}(z) \varphi_{i}(z)\right),
$$

is continuous. If we put $k_{i}(z)=\sum_{j=1}^{n} a_{j i}(z) \varphi_{j}(z)$, then for every $z \in \mathcal{O}$, we get

$$
N\left(T^{*}-z\right)=\operatorname{span}\left\{k_{1}(z), k_{2}(z), \ldots, k_{n}(z)\right\} \quad \text { and } \quad\left\langle y_{i}, k_{j}(z)\right\rangle=\delta_{i j}, \forall i, j,
$$

and hence $\mathcal{O} \subset \mathcal{B}_{a}(T)$.

Combining Theorem 4 and Theorem 5, we derive

Corollary 1. The sets $\mathcal{B}(T)$ and $\mathcal{B}_{a}(T)$ do not depend on the choice of the cyclic $n$ tuple.

By using Baire's category theorem and the characterization of $\mathcal{B}_{a}(T)$ given by Proposition 3 , we obtain easily (see $[28,14]$ )

$$
\mathcal{B}_{a}(T) \subset \operatorname{int}(\mathcal{B}(T)) \subset \operatorname{cl}(\mathcal{B}(T)) .
$$

It is not clear whether the following inclusions hold for $m<n$ :

$$
\mathcal{B}_{a}^{m}(T) \subset \operatorname{int}\left(\mathcal{B}^{m}(T)\right) \subset \operatorname{cl}\left(\mathcal{B}^{m}(T)\right)
$$

To obtain (14), one has to provide a version of Proposition 3 for $m<n$.

We associate with $T$ the following (not necessarily closed) linear subspace of $\mathcal{X}$ : The analytic core $K(T)$ of $T$ is defined to be $K(T)=\left\{u \in H ; \exists c>0\right.$ and $\left(v_{n}\right)_{n \geq 0} \in H$ such that (1) $u=v_{0}$ and $\left.v_{n}=T v_{n+1},(2)\left\|v_{n}\right\| \leq c^{n}\|u\|\right\}$. A systematic investigation of $K(T)$ was initiated by the second named author in [17]. If $E$ is any closed invariant subspace for $T$ such that $T(E)=E$, then $E \subset K(T)$. In particular if $T$ is onto, then $K(T)=\mathcal{X}$. We refer to $[17,18]$ for further properties of the analytic core.

We link the analytic core and the analytic structures as follows:

THEOREM 6. Let $T$ be an $n$-multicyclic operator and $1 \leq m \leq n$. We have

i) If $m \neq n$, then

$$
\begin{aligned}
\mathcal{B}_{a}^{m}(T) & =\left\{\lambda \in \operatorname{int}\left(\mathcal{B}^{m}(T)\right) ; \quad \operatorname{dim}\left(N(T-\lambda)^{*} \cap K(T-\lambda)^{*}\right)=m\right\} \\
& =\left\{\lambda \in \operatorname{int}\left(\mathcal{B}^{m}(T)\right) ; \quad N(T-\lambda)^{*} \subset K(T-\lambda)^{*}\right\} .
\end{aligned}
$$

ii)

$$
\begin{aligned}
\mathcal{B}_{a}(T) & =\left\{\lambda \in \mathcal{B}(T) ; \quad \operatorname{dim}\left(N(T-\lambda)^{*} \cap K(T-\lambda)^{*}\right)=n\right\} \\
& =\left\{\lambda \in \mathcal{B}(T) ; \quad N(T-\lambda)^{*} \subset K(T-\lambda)^{*}\right\} .
\end{aligned}
$$

Proof. $i$ ) Suppose that $0 \in \mathcal{B}_{a}^{m}(T)$ and let $k_{1}, k_{2}, \ldots, k_{m}$ be $\mathcal{X}$-valued analytic functions defined on some neighbourhood $\mathcal{V}$ of zero and such that $N(T-\lambda)^{*}=\operatorname{span}\left\{k_{1}(\lambda), k_{2}(\lambda), \ldots\right.$, $\left.k_{m}(\lambda)\right\}$ for every $\lambda \in \mathcal{V}$. By writing $k_{i}(\lambda)=\sum_{n=0}^{\infty} a_{i, n} \lambda^{n}$ the Taylor expansion of $k_{i}$ at zero, we deduce that $a_{i, 0}=k_{i}(0),(T-\lambda)^{*} a_{i+1, n}=a_{i, n}$ and $\left|a_{i, n}\right| \leq c^{n}$ for some non-zero constant. Thus $k_{1}, k_{2}, \ldots, k_{m} \in K(T-\lambda)^{*}$ and the first inclusion is obtained.

It remains to show that $\left\{\lambda \in \operatorname{int}\left(\mathcal{B}^{m}(T)\right) ; \operatorname{dim}\left(N(T-\lambda)^{*} \cap K(T-\lambda)^{*}\right)=m\right\} \subset \mathcal{B}_{a}^{m}(T)$. To this end suppose that $\operatorname{dim}\left(N(T)^{*} \cap K(T)^{*}\right)=m$. We will show that $0 \in \mathcal{B}_{a}^{m}(T)$. Let $k_{1}, k_{2}, \ldots, k_{m}$ be a basis of $\left.N(T)^{*} \cap K(T)^{*}\right)$ and let $a_{i, n}$ be a family of vectors in $\mathcal{X}$ given 
by the definition of $K\left(T^{*}\right)$ and such that $k_{i}=a_{i, 0}$. Set now $k_{i}(\lambda)=\sum_{n=0}^{\infty} a_{i, n} \lambda^{n}$; then $k_{1}, k_{2}, \ldots, k_{m}$ are a linearly independent family of $\mathcal{X}$-valued analytic functions defined on some neighbourhood of zero and satisfy $\operatorname{span}\left\{k_{1}(\lambda), k_{2}(\lambda), \ldots, k_{m}(\lambda)\right\} \subset N(T-\lambda)^{*}$. Now since $0 \in \operatorname{int}\left(\mathcal{B}^{m}(T)\right)$, we have $\operatorname{dim}\left(N(T-\lambda)^{*}\right)=m$ in some neighbourhood of zero and we conclude that $\operatorname{span}\left\{k_{1}(\lambda), k_{2}(\lambda), \ldots, k_{m}(\lambda)\right\}=N(T-\lambda)^{*}$. Finally $0 \in \mathcal{B}_{a}^{m}(T)$. The second equality in $i$ ) is trivial.

ii) The proof goes similarly to $i)$, the only difference is that since $\operatorname{dim}\left(N(T-\lambda)^{*}\right) \leq n$ for every $\lambda \in \mathbb{C}$, the inclusion $\operatorname{span}\left\{k_{1}(\lambda), k_{2}(\lambda), \ldots, k_{n}(\lambda)\right\} \subset N(T-\lambda)^{*}$ leads to the equality $\operatorname{span}\left\{k_{1}(\lambda), k_{2}(\lambda), \ldots, k_{n}(\lambda)\right\}=N(T-\lambda)^{*}$ and hence to the conclusion $0 \in$ $\mathcal{B}_{a}(T)$.

We mention here that the interior cannot be removed from the equality given in Theorem $6 i$ ) as shown by Example 2 below. Note also that Theorem 6 is given in [22] for cyclic operators.

REMARK 4. 1. In $[11,13]$ D. A. Herrero introduced the concept of the analytic structure of an $n$-multicyclic operator. More precisely, an open set $O$ is said to be contained in the analytic structure of $T$ if there exists a family of analytic functions $\phi_{i}, i=1, \ldots, n$ on $O$ such that $N\left(T^{*}-z\right)=\operatorname{span}\left\{\phi_{1}(z), \phi_{2}(z), \ldots, \phi_{i}(z)\right\}$ every $z \in O$. As shown by Theorem 5 , if $T$ is an $n$-cyclic operator, the analytic structure in the sense of [13] and the set of analytic bounded point evaluations of $T$ coincide.

2. Let $T$ be a bounded operator, and let $\lambda_{0}$ be a complex number. Following J. Finch [9], we say that the operator $T$ has the single valued extension property at $\lambda_{0}((S V E P)$, for short) if there exists $\delta>0$ such that $f \equiv 0$ is the only solution to the equation $(T-\lambda) f(\lambda)=0$ that is analytic in $D\left(\lambda_{0}, \delta\right)$. Also, $T$ has the single valued extension property if it has this property at every $\lambda_{0}$ in the complex plane. Let $\mathcal{A}(T)$ be the set of all complex numbers where $T$ has the $(S V E P)$ and $\mathcal{S}(T)=\mathbb{C} \backslash \mathcal{A}(T)$. Then clearly $\lambda_{0} \in \mathcal{S}(T)$ if and only if there exists $\delta>0$ and a nonzero analytic function $f$ in $D\left(\lambda_{0}, \delta\right)$ satisfying $(T-\lambda) f(\lambda)=0$. In particular $\mathcal{S}(T)$ is open and $T$ has the $(S V E P)$ if and only if $\mathcal{S}(T)=\emptyset$.

It is not hard to see that in the case of cyclic operators, $\mathcal{S}\left(T^{*}\right)=\mathcal{B}_{a}(T)$. To get the same equality for multicyclic operators seems to be reasonable. However, in view of Example 2 below and Theorem 4 this is not true.

An analogous spectral subset is given by the residual spectrum. The residual spectrum $S_{T}$ of $T$ is defined as the complement in $\mathbb{C}$ of the largest open set $O$ such that, if $f$ is any analytic function in an arbitrary open subset $V$ of $O$ satisfying the equation $(T-\lambda) f(\lambda)=0$, then $f \equiv 0$ (see [32, Ch. IV]). Clearly the residual spectrum is closed and $T$ has the $(S V E P)$ precisely when $S_{T}=\emptyset$. Moreover

$$
S_{T^{*}}=\operatorname{cl}\left(\mathcal{S}\left(T^{*}\right)\right)=\operatorname{cl}\left(\mathcal{B}_{a}(T)\right) .
$$

The inclusion $\operatorname{cl}\left(\bigcup_{m=1}^{n} \mathcal{B}_{a}^{m}(T)\right) \subseteq S_{T^{*}}$ holds for arbitrary multicyclic operators. In the example below this inclusion turns out to be an equality. Therefore, we pose the question. Is $\operatorname{cl}\left(\bigcup_{m=1}^{n} \mathcal{B}_{a}^{m}(T)\right)=S_{T^{*}}$ for every multicyclic operator?

ExAmple 2. Let $S$ be the shift operator defined on the Hardy space $H^{2}$ and set $\mathcal{X}=$ $H^{2} \oplus H^{2}$. For $a_{1}, a_{2}, b_{1}$ and $b_{2}$ non-vanishing complex numbers, consider the operator $T=T_{1} \oplus T_{2}$ with $T_{i}=a_{i} I+b_{i} S$, for $i=1,2$. 
We claim that if $0<\left|a_{1}-a_{2}\right|<\left|b_{1}\right|+\left|b_{2}\right|$ then $T$ is 2-multicyclic. Indeed, clearly we have $D\left(a_{i},\left|b_{i}\right|\right) \subset \sigma_{p}\left(T_{i}^{*}\right)$ and $\sigma(T) \subset \bar{D}\left(a_{i},\left|b_{i}\right|\right)$. For $i=1,2$, the condition $0<\left|a_{1}-a_{2}\right|<$ $\left|b_{1}\right|+\left|b_{2}\right|$ implies that $D\left(a_{1},\left|b_{1}\right|\right) \cap D\left(a_{2},\left|b_{2}\right|\right) \neq \emptyset$ and for $\lambda \in D\left(a_{1},\left|b_{1}\right|\right) \cap D\left(a_{2},\left|b_{2}\right|\right)$ we have $\operatorname{dim} N(T-\lambda)^{*}=2$. Now since $T_{1}$ and $T_{2}$ are cyclic operators, the operator $T$ is 2-multicyclic.

The following equalities are straightforward:

1. $\operatorname{int}\left(\mathcal{B}\left(T_{i}\right)\right)=\mathcal{B}_{a}\left(T_{i}\right)=D\left(a_{i},\left|b_{i}\right|\right)$, for $i=1,2$.

2. $\mathcal{B}_{a}(T)=\mathcal{B}_{a}^{2}(T)=D\left(a_{1},\left|b_{1}\right|\right) \cap D\left(a_{2},\left|b_{2}\right|\right)$.

3. $\mathcal{B}_{a}^{1}(T)=D\left(a_{1},\left|b_{1}\right|\right) \backslash \bar{D}\left(a_{i},\left|b_{i}\right|\right) \cup D\left(a_{2},\left|b_{2}\right|\right) \backslash \bar{D}\left(a_{i},\left|b_{i}\right|\right)$.

4. $\mathcal{S}\left(T^{*}\right)=D\left(a_{1},\left|b_{1}\right|\right) \cup D\left(a_{2},\left|b_{2}\right|\right)$.

5. $\mathcal{B}^{1}(T)=D\left(a_{1},\left|b_{1}\right|\right) \backslash D\left(a_{i},\left|b_{i}\right|\right) \cup D\left(a_{2},\left|b_{2}\right|\right) \backslash D\left(a_{i},\left|b_{i}\right|\right)$.

6. (a) $K(T-\lambda)=H$ for every $\lambda \in \mathcal{B}_{a}(T)$.

(b) $K(T-\lambda)=H^{2} \oplus\{0\}$ for $\lambda \in \mathcal{B}_{a}\left(T_{1}\right)$ and $K(T-\lambda)=\{0\} \oplus H^{2}$ for $\lambda \in \mathcal{B}_{a}\left(T_{2}\right)$.

Thus, we have

i) $\mathcal{B}_{a}(T) \neq \mathcal{S}\left(T^{*}\right)$.

ii) $\operatorname{cl}\left(\bigcup_{m=1}^{n} \mathcal{B}_{a}^{m}(T)\right)=S_{T^{*}}$

iii) $\mathcal{B}_{a}^{1}(T) \neq\left\{\lambda \in \mathcal{B}^{1}(T) ; \operatorname{dim}\left(N(T-\lambda)^{*} \cap K(T-\lambda)^{*}\right)=1\right\}$.

4. Analytic structures and the singular spectrum. An operator $T \in \mathcal{L}(\mathcal{X})$ is said to be semi-regular if $R(T)$ is closed and if for every $n \geq 0$, we have $N\left(T^{n}\right) \subseteq R(T)$. The semi-regular resolvent set $\mathrm{s}-\mathrm{reg}(T)$ is

$$
\text { s-reg }(\mathrm{T})=\{\lambda \in \mathbb{C} ; T-\lambda \text { est semi-regular }\} .
$$

The complement of s-reg $(T)$ in $\mathbb{C}$ is called the singular spectrum and is denoted by $\sigma_{s}(T)$.

The singular spectrum appears in the literature and takes many other names (Kato spectrum, Apostol spectrum,..., see [14, 23, 24]). We assemble in the following remark some important properties of the singular spectrum.

REMARK 5. 1) $\sigma_{s}(T)$ is a compact subset of $\sigma(T)$.

2) $\partial \sigma(T) \subseteq \sigma_{s}(T) \subseteq \sigma_{a p}(T) \subseteq \sigma(T)$.

3) $\sigma_{s}(T)=\sigma_{s}\left(T^{*}\right)$.

4) The spectral mapping theorem holds for the singular spectrum in the following sense. If $f$ is any analytic function in a neighbourhood of $\sigma(T)$, then $\sigma_{s}(f(T))=f\left(\sigma_{s}(T)\right)$.

5) The mapping $\lambda \mapsto \operatorname{dim} N(T-\lambda)$ is constant in connected components of $\operatorname{s-reg}(T)$.

In the following theorem from [19], we find a useful characterization of $\mathrm{s}-\mathrm{reg}(T)$. We refer to [19] for the proof.

TheOREM 7 ([19, Théorème 2.7]). Let $T \in \mathcal{L}(\mathcal{X})$ be a bounded operator and suppose that $R(T)$ is closed. Then the following are equivalent:

i) $0 \in \operatorname{s-reg}(T)$;

ii) For every $x \in N(T)$ there exist a neighbourhood $U$ of zero and an analytic function $f: U \rightarrow \mathcal{X}$ such that $f(0)=x$ and $f(\lambda) \in N(T-\lambda)$ for every $\lambda \in U$. 
A direct consequence of Theorem 7 is:

Corollary 2. Let $T \in \mathcal{L}(\mathcal{X})$. Then

$$
\mathbb{C} \backslash \sigma_{a p}(T)=s-\operatorname{reg}(T) \cap \beta(T)=s-\operatorname{reg}(T) \cap \mathcal{A}(T) .
$$

In particular if $T$ has the $(S V E P)$ then $\sigma_{s}(T)=\sigma_{a p}(T)$.

The reader is referred to section 5 for the definition of $\beta(T)$.

If $A$ is a matrix, denote by $\operatorname{rank}(A)$ its rank. The following lemma generalizes Lemma 1 and is central in the study of analytic structures.

Lemma 2. Let $T \in \mathcal{C}_{n}(\mathcal{X}),\left(y_{1}, \ldots, y_{n}\right) \in C_{n}(T)$ and let $\lambda$ be a complex number. If $N(T-\lambda)^{*}=\operatorname{span}\left\{k_{1}, k_{2}, \ldots, k_{n}\right\}$ then

$$
\operatorname{rank}\left(\left\langle y_{i}, k_{j}\right\rangle_{i j}\right)=\operatorname{dim}\left(N(T-\lambda)^{*}\right) .
$$

Proof. It is obvious that $\operatorname{rank}\left(\left\langle y_{i}, k_{j}\right\rangle_{i j}\right) \leq \operatorname{dim} N(T-\lambda)^{*}$. To see the reverse inequality, if $\operatorname{rank}\left(\left\langle y_{i}, k_{j}\right\rangle_{i j}\right)=m$, then there exists an invertible matrix $A$ such that $H(\lambda) A$ has the following form:

$$
H(\lambda) A=\left(\begin{array}{cccccc}
a_{11} & \ldots & a_{1 m} & 0 & \ldots & 0 \\
a_{21} & \ldots & a_{2 m} & 0 & \ldots & 0 \\
\vdots & \vdots & \vdots & \vdots & \vdots & \vdots \\
a_{n 1} & \ldots & a_{n m} & 0 & \ldots & 0
\end{array}\right)
$$

Hence, there exist $h_{1}, h_{2}, \ldots, h_{m}$ such that $N(T-\lambda)^{*}=\operatorname{span}\left\{h_{1}, h_{2}, \ldots, h_{m}\right\}$ and

$$
H(\lambda) A=\left(\begin{array}{cccccc}
\left\langle y_{1}, h_{1}\right\rangle & \ldots & \left\langle y_{1}, h_{m}\right\rangle & 0 & \ldots & 0 \\
\left\langle y_{2}, h_{1}\right\rangle & \ldots & \left\langle y_{2}, h_{m}\right\rangle & 0 & \ldots & 0 \\
\vdots & \vdots & \vdots & \vdots & \vdots & \vdots \\
\left\langle y_{n}, h_{1}\right\rangle & \ldots & \left\langle y_{n}, h_{m}\right\rangle & 0 & \ldots & 0
\end{array}\right) .
$$

Consequently $\operatorname{dim}\left(N(T-\lambda)^{*}\right) \leq m$. The proof is complete.

Proposition 4. Let $T$ be an $n$-multicyclic operator defined on a Banach space $\mathcal{X}$ and $1 \leq m \leq n$. Let $G$ be a connected component of $s$-reg $(T)$ such that $G \cap \mathcal{B}^{m}(T) \neq \emptyset$. Then $G \subseteq \mathcal{B}_{a}^{m}(T)$. In particular if $G \cap \mathcal{B}(T) \neq \emptyset$, then $G \subseteq \mathcal{B}_{a}(T)$.

Proof. Since $G \cap \mathcal{B}^{m}(T) \neq \emptyset$ and since $\operatorname{dim} N(T-z)^{*}$ is constant in connected components of s-reg $(T)$, we get $G \subseteq \mathcal{B}^{m}(T)$.

Suppose now that $0 \in G \cap \mathcal{B}^{m}(T)$ and let $\left\{k_{1}, k_{2}, \ldots, k_{m}\right\}$ be a family of vectors in $\mathcal{X}$ such that $N(T)^{*}=\operatorname{span}\left\{k_{1}, k_{2}, \ldots, k_{m}\right\} \quad\left(G \subseteq \operatorname{s-reg}(T)=\operatorname{s}-\operatorname{reg}\left(T^{*}\right)\right)$. Then by Theorem 7, there exists an open neighbourhood $\Omega \subseteq G$ of 0 and there are analytic functions $\varphi_{i}: \mathcal{O} \rightarrow \mathcal{X}$ such that $k_{i}=\varphi_{i}(0)$ and $(T-z)^{*} \varphi_{i}(z)=0$, for every $z \in \Omega$ and for every $i \in\{1,2, \ldots, m\}$.

For $z \in \Omega$, consider the matrix

$$
H(z)=\left(\begin{array}{cccccc}
\left\langle y_{1}, \varphi_{1}(z)\right\rangle & \ldots & \left\langle y_{1}, \varphi_{m}(z)\right\rangle & 0 & \ldots & 0 \\
\left\langle y_{2}, \varphi_{1}(z)\right\rangle & \ldots & \left\langle y_{2}, \varphi_{m}(z)\right\rangle & 0 & \ldots & 0 \\
\vdots & \vdots & \vdots & \vdots & \vdots & \vdots \\
\left\langle y_{n}, \varphi_{1}(z)\right\rangle & \ldots & \left\langle y_{n}, \varphi_{m}(z)\right\rangle & 0 & \ldots & 0
\end{array}\right)
$$


Since the functions $\varphi_{i}$ are analytic, the mapping $z \in \Omega \mapsto r g(H(z))$ is lower semicontinuous. On the other hand by Lemma 2 we get $\operatorname{rank}(H(0))=\operatorname{dim} N\left(T^{*}\right)=m$, which implies that for some neighbourhood $\mathcal{V}$ of 0 contained in $G$, we have $\operatorname{rank}(H(z))=m$, for every $z \in \mathcal{V}$. Consequently, $\mathcal{V} \subseteq \mathcal{B}_{a}^{m}(T)$. This completes the proof of Proposition 4 .

The following corollary is a direct application of the preceding proposition and the inclusion $\sigma_{s}(T) \subseteq \sigma_{a p}(T)$.

Corollary 3. Let $T$ be an $n$-multicyclic operator and $m \in\{1,2, \ldots, n\}$. Then

$$
\mathcal{B}^{m}(T) \backslash \sigma_{a p}(T) \subseteq \mathcal{B}^{m}(T) \backslash \sigma_{s}(T) \subseteq \mathcal{B}_{a}^{m}(T) .
$$

In particular,

$$
\mathcal{B}(T) \backslash \sigma_{a p}(T) \subseteq \mathcal{B}(T) \backslash \sigma_{s}(T) \subseteq \mathcal{B}_{a}(T) .
$$

It is observed in [21] that none of the preceding inclusions can be reversed in general.

An operator $T \in \mathcal{L}(\mathcal{X})$ is a Fredholm operator if $R(T)$ is closed and $\max (\operatorname{dim}(N(T))$, $\operatorname{codim}(R(T)))$ is finite. The operator $T$ is semi-Fredholm if $R(T)$ is closed and $\min (\operatorname{dim}(N(T)), \operatorname{codim}(R(T)))$ is finite. The semi-Fredholm spectrum of $T$ is $\sigma_{s-F}(T)=$ $\{\lambda \in \mathbb{C} ; T-\lambda$ is not semi-Fredholm $\}$. The index of a semi-Fredholm operator $T$ is defined to be $\operatorname{ind}(T)=\operatorname{dim}(N(T))-\operatorname{codim}(R(T))$. Since $n$-multicyclic operators satisfy $\operatorname{codim}(R(T-\lambda)) \leq n$ for every $\lambda \in \mathbb{C}$, we get that $T-\lambda$ is semi-Fredholm if and only if $R(T-\lambda)$ is closed, moreover ind $(T-\lambda) \geq-n$ for every $\lambda \in \mathbb{C}$. If we denote $\sigma_{f}(T)=\{\lambda \in \mathbb{C} ; R(T-\lambda)$ is not closed $\}$, then it follows from the discussion above that $\sigma_{s-F}(T)=\sigma_{f}(T)$.

Corollary 4. Let $T$ be an $n$-multicyclic operator and $m \in\{1,2, \ldots, n\}$. Then

$$
\mathcal{B}^{m}(T) \backslash \sigma_{s}(T)=\mathcal{B}_{a}^{m}(T) \backslash \sigma_{s-F}(T) .
$$

In particular,

$$
\mathcal{B}(T) \backslash \sigma_{s}(T)=\mathcal{B}_{a}(T) \backslash \sigma_{s-F}(T) .
$$

Moreover, if $T$ has the (SVEP) then

$$
\mathcal{B}(T) \backslash \sigma_{a p}(T)=\mathcal{B}_{a}(T) \backslash \sigma_{s-F}(T) .
$$

Proof. Because of Corollary 3, we only have to prove the reverse inclusion. Suppose that $0 \in B_{m}^{a}(T)$ and that $R(T)$ is closed. Then $R\left(T^{*}\right)$ is closed. Now apply Theorem 7 with $T^{*}$ to end the proof.

For $k \in \mathbb{Z}$, set $\rho_{s-F}^{k}(T)=\left\{\lambda \in \rho_{s-F}(T) ; i n d(T-\lambda)=k\right\}$. In [11] D. A. Herrero has proved that, if $T$ is an $n$-multicyclic operator on some Banach space, then $\rho_{s-F}^{-n}(T)=$ $\left\{\lambda \in \rho_{s-F}(T) ; \operatorname{dim} N(T-\lambda)=0\right.$ and $\left.\operatorname{dim} N(T-\lambda)^{*}=n\right\}$. In particular $\rho_{s-F}^{-n}(T) \subset$ $\mathcal{B}(T) \cap s-\operatorname{reg}(T)$. Hence $\rho_{s-F}^{-n}(T) \subset \mathcal{B}_{a}(T)$. It is also shown that connected components of $\rho_{s-F}^{-n}(T)$ are actually simply connected (see [11, Theorem 1]). The following proposition provides the same conclusion for connected components of $\mathcal{B}_{a}(T)$.

Proposition 5. Connected components of $\mathcal{B}_{a}(T)$ are simply connected.

Proof. Let $\gamma$ be a simple Jordan curve and denote by ins $(\gamma)$ the bounded component of $\mathbb{C} \backslash \gamma$. To see that connected components of $\mathcal{B}_{a}(T)$ are actually simply connected, it suffices to show that for every simple Jordan curve $\gamma$ in $\mathcal{B}_{a}(T)$, we have $\operatorname{ins}(\gamma) \subset \mathcal{B}_{a}(T)$. 
Let now $\gamma$ be such a Jordan curve in $\mathcal{B}_{a}(T)$. Since $\gamma$ is a compact subset of $\mathcal{B}_{a}(T)$, there exists $M>0$ such that

$$
\sup _{1 \leq i \leq n}\left\{\left\|k_{i}(\lambda)\right\|, \lambda \in \gamma\right\} \leq M
$$

Then for any polynomials $P_{1}, P_{2}, \ldots, P_{n}$ in $\mathcal{P}$ and every $\lambda \in \gamma$, we have

$$
\left|P_{j}(\lambda)\right| \leq \sum_{i=1}^{n}\left|P_{i}(\lambda)\right| \leq M\left\|\sum_{i=1}^{n} P_{i}(T) y_{i}\right\|, \quad \text { for every } j=1,2, \ldots, n .
$$

The maximum modulus principle gives

$$
\sum_{i=1}^{n}\left|P_{i}(\lambda)\right| \leq n M\left\|\sum_{i=1}^{n} P_{i}(T) y_{i}\right\|, \quad \text { for every } \lambda \in \text { ins }(\gamma) .
$$

Thus ins $(\gamma) \subset \mathcal{B}(T)$ and $\sup _{1 \leq i \leq n}\left\{\left\|k_{i}(\lambda)\right\|, \lambda \in\right.$ ins $\left.(\gamma)\right\} \leq M$. Now, we conclude by using Proposition 3.

REMARK 6. Connected components of $\mathcal{B}_{a}^{m}(T)$, for $1 \leq m \leq n-1$, need not be simply connected. To see this, let $S$ be the usual shift operator defined on $H^{2}$ and $T=S \oplus 2 S$ defined on $H^{2} \oplus H^{2}$. Then $T$ is 2-multicyclic and $\mathcal{B}_{a}^{1}=\{z \in \mathbb{C} ; 1<|z|<2\}$, which is clearly not simply connected.

5. Bishop's property $(\beta)$ and bounded point evaluations. Recall that an operator $T \in \mathcal{L}(\mathcal{X})$ is said to possess Bishop's property $(\beta)$ at $\lambda \in \mathbb{C}$ (we will also say for simplicity $T$ has $(\beta)$ at $\lambda)$ if there exists $\delta>0$ such that for every open set $\Omega$ contained in $D(\lambda, \delta)$, and any sequence $\left(f_{n}\right)_{n \geq 1}$ in $\mathcal{H}(\Omega, H)$, the convergence of the sequence $(T-z) f_{n}(z)$ to zero in $\mathcal{H}(\Omega, H)$ entails the convergence of $f_{n}(z)$ to zero in $\mathcal{H}(\Omega, H)$. We shall denote by $\beta(T)$ the open set of all complex numbers where $T$ possesses property $(\beta)$, and $\sigma_{\beta}(T)$ its complement in $\mathbb{C}$. We say that $T$ has $(\beta)$ when $\beta(T)=\mathbb{C}$. It is clear from the definition that if $T$ has $(\beta)$ then $T$ satisfies the $(S V E P)$.

A nice characterization of operators with $(\beta)$ is given by E. Albrecht and J. Eschmeier in [2]. Namely, an operator $T$ has $(\beta)$ precisely when it has a decomposable extension. In particular all subnormal operators have $(\beta)$. We refer to the monograph [14] for further details and definitions.

TheOREM 8. Let $T$ be an $n$-multicyclic operator defined on a Banach space $\mathcal{X}$. Suppose that $T$ has $(\beta)$. Then

$$
\mathcal{B}_{a}(T)=\mathcal{B}(T) \backslash \sigma_{a p}(T) .
$$

Theorem 8 follows from the fact that under the assumption that $T$ has $(\beta)$ we have $\sigma_{a p}(T)=\sigma_{s}(T)$ and from the following more general theorem.

TheOREM 9. Let $T \in \mathcal{L}(\mathcal{X})$ be an $n$-multicyclic operator. Then

$$
\mathcal{B}(T) \backslash \sigma_{a p}(T)=\mathcal{B}_{a}(T) \backslash \sigma_{\beta}(T) .
$$

Proof. The inclusion $\mathcal{B}(T) \backslash \sigma_{a p}(T) \subset \mathcal{B}_{a}(T) \backslash \sigma_{\beta}(T)$ is obtained from Corollary 2 and Proposition 2. Suppose now that $0 \in \mathcal{B}_{a}(T) \backslash \sigma_{\beta}(T)$. Then $R(T)$ is closed. Indeed, let $y \in \mathcal{H}$ and let $\left(x_{m}\right)_{m \geq 1}$ be a sequence of elements in $\mathcal{X}$ such that $x_{m}=\sum_{i=1}^{n} P_{i, m}(T) y_{i}$ 
and $\lim _{n \rightarrow \infty}\left\|x-x_{m}\right\|=0$. For $1 \leq i \leq n$ and $m \geq 1$, consider the analytic function defined in a neighbourhood $\mathcal{V}$ of 0 by

$$
y_{m}(z):=\sum_{i=1}^{n} P_{i, m}(T) y_{i}-\sum_{i=1}^{n}\left\langle P_{i, m}(T) y_{i}, k_{i}(z)\right\rangle y_{i} .
$$

Then $\lim _{m \rightarrow \infty} y_{m}(z)=y-\sum_{i=1}^{n}\left\langle y, k_{i}(z)\right\rangle y_{i}$. On the other hand,

$$
\begin{aligned}
y_{m}(z) & =\sum_{i=1}^{n} P_{i, m}(T) y_{i}-\sum_{i=1}^{n} P_{i, m}(z) y_{i}=\sum_{i=1}^{n}\left[P_{i, m}(T)-P_{i, m}(z)\right] y_{i} \\
& =(T-z) \sum_{i=1}^{n} Q_{i, m}(T, z) y_{i}
\end{aligned}
$$

where $Q_{i, m}(., z)$ is the complex polynomial given by $Q_{i, m}(t, z)=\frac{P_{i, m}(z)-P_{i, m}(t)}{z-t}$. The mapping $z \mapsto Q_{i, m}(T, z)$ is analytic at $0 \in \beta(T)$, and we get

$$
\lim _{m \rightarrow \infty}(T-z) \sum_{i=1}^{n} Q_{i, m}(T, z) \in \mathcal{R}(T-z),
$$

for every $z \in \mathcal{V}$.

In particular for $z=0$, there exists $x \in \mathcal{X}$ such that

$$
y=\sum_{i=1}^{n}\left\langle y, k_{i}(0)\right\rangle y_{i}+T x .
$$

Thus, $\operatorname{codim} R(T) \leq n$ and hence $R(T)$ is closed. Thus $0 \in \mathcal{B}_{a}(T) \cap s-\operatorname{reg}(T)$, and consequently $0 \in \beta(T) \cap s-\operatorname{reg}(T)=\mathbb{C} \backslash \sigma_{a p}(T)$.

We end this section by posing the following question. Does Equality (16) below hold for arbitrary $n$-multicyclic operators and $0 \leq m \leq n$ ?

$$
\mathcal{B}^{m}(T) \backslash \sigma_{a p}(T)=\mathcal{B}_{a}^{m}(T) \backslash \sigma_{\beta}(T) .
$$

6. On the density of $\mathcal{C}_{n}(T)$. Let $T \in \mathcal{C}_{n}(\mathcal{X})$ be $n$-multicyclic. We retrieve in this section a theorem of D. A. Herrero and L. Rodman [13]. We present here a proof depending on analytic bounded point evaluations that is much simpler.

TheOREM 10. Let $T$ be an n-multicyclic operator defined on a Banach space $\mathcal{X}$. The following assertions are equivalent:

(i) $\mathcal{B}_{a}(T) \neq \emptyset$.

(ii) $\mathcal{C}_{n}(T)$ is not dense in $\mathcal{X}^{(n)}$.

To prove Theorem 10, we start with some results of independent interest. We describe the bounded point evaluations set and the analytic bounded point evaluations set in terms of cyclic $n$-tuples.

Proposition 6. Let $T$ be an $n$-multicyclic operator and $\left(y_{1}, y_{2}, \ldots, y_{n}\right) \in \mathcal{C}_{n}(T)$. Then

(i) $\mathcal{B}(T)=\left\{\lambda \in \mathbb{C} ; \quad\left((T-\lambda) y_{1}, y_{2}, \ldots, y_{n}\right) \notin \mathcal{C}_{n}(T)\right\}$.

(ii) $\mathcal{B}_{a}(T) \subset\left\{\lambda \in \mathbb{C} ;\left((T-\lambda) y_{1}, y_{2}, \ldots, y_{n}\right) \notin \operatorname{cl}\left(\mathcal{C}_{n}(T)\right)\right\} \subset \operatorname{int}(\mathcal{B}(T))$. 
Proof. (i) If $\lambda \in \mathbb{C}$ is such that $\left((T-\lambda) y_{1}, y_{2}, \ldots, y_{n}\right) \in \mathcal{C}_{n}(T)$, then

$$
\begin{aligned}
\mathcal{X} & =\operatorname{span}\left\{T^{m}(T-\lambda) y_{1}, T^{m} y_{2}, \ldots, T^{m} y_{n}, m \geq 0\right\} \\
& =\operatorname{span}\left\{T^{m}(T-\lambda) y_{1},\left(T^{m}-\lambda^{m}\right) y_{2}, \ldots,\left(T^{m}-\lambda^{m}\right) y_{n}, y_{2}, \ldots, y_{n} ; m \geq 1\right\} \\
& \subset \operatorname{cl}(\mathcal{R}(T-\lambda))+\operatorname{span}\left\{y_{2}, \ldots, y_{n}\right\} .
\end{aligned}
$$

Then $\operatorname{dim} N(T-\lambda)^{*}<n$ and hence $\lambda \notin \mathcal{B}(T)$.

Conversely suppose that $\operatorname{dim} N(T-\lambda)^{*}<n$. By Proposition 2, we get $\operatorname{cl}(\mathcal{R}(T-\lambda))$ $\cap \operatorname{span}\left\{y_{1}, y_{2}, \ldots, y_{n}\right\} \neq\{0\}$. Replacing if necessary $\left\{y_{1}, y_{2}, \ldots, y_{n}\right\}$ by another basis of $\operatorname{span}\left\{y_{1}, y_{2}, \ldots, y_{n}\right\}$, we may suppose without loss of generality that $y_{1} \in \operatorname{cl}(\mathcal{R}(T-\lambda))$. Thus

$$
\begin{aligned}
\mathcal{X} & =\operatorname{cl}(\mathcal{R}(T-\lambda))+\operatorname{span}\left\{y_{2}, \ldots, y_{n}\right\} \\
& \subset \operatorname{span}\left\{T^{k}(T-\lambda) y_{1}, T^{k} y_{2}, \ldots, T^{k} y_{n} ; k \geq 0\right\} .
\end{aligned}
$$

And hence $\left((T-\lambda) y_{1}, y_{2}, \ldots, y_{n}\right) \in \mathcal{C}_{n}(T)$.

(ii) The inclusion $\left\{\lambda \in \mathbb{C} ;\left((T-\lambda) y_{1}, y_{2}, \ldots, y_{n}\right) \notin \operatorname{cl}\left(\mathcal{C}_{n}(T)\right)\right\} \subset \operatorname{int}(\mathcal{B}(T))$ follows from $(i)$ by using standard arguments of continuity. It remains to show that $\mathcal{B}_{a}(T) \subset$ $\left\{\lambda \in \mathbb{C} ;\left((T-\lambda) y_{1}, y_{2}, \ldots, y_{n}\right) \notin \operatorname{cl}\left(\mathcal{C}_{n}(T)\right)\right\}$. Let $\lambda \in \mathcal{B}_{a}(T)$ and $r>0$ be such that $\bar{D}(\lambda, r) \subset \mathcal{B}_{a}(T)$. Set

$$
\Omega(\lambda)=\left\{\left(x_{1}, x_{2}, \ldots, x_{n}\right) \in \mathcal{X}^{n} ; \sup _{|z|=r}\left|z-\lambda-\operatorname{det}\left(\left\langle x_{i}, k_{j}(z)\right\rangle\right)_{i, j}\right|<r\right\} .
$$

Then $\Omega(\lambda)$ is a nonempty open subset of $\mathcal{X}^{(n)}$ and clearly $\left.(T-\lambda) y_{1}, y_{2}, \ldots, y_{n}\right) \in \Omega(\lambda)$. We claim that $\Omega(\lambda) \cap \mathcal{C}_{n}(T)=\emptyset$. Indeed, suppose that there exists $\left(x_{1}, x_{2}, \ldots, x_{n}\right) \in$ $\Omega(\lambda) \cap \mathcal{C}_{n}(T)$. Since $\left(x_{1}, x_{2}, \ldots, x_{n}\right) \in \mathcal{C}_{n}(T)$ we have $\operatorname{det}\left(\left\langle x_{i}, k_{j}(z)\right\rangle_{i, j}\right) \neq 0$, for every $z \in \mathcal{B}(T)$. Consider now the following continuous function:

$$
f: \bar{D}(\lambda, r) \rightarrow \bar{D}(\lambda, r), \quad z \mapsto z-\operatorname{det}\left(\left\langle x_{i}, k_{j}(z)\right\rangle_{i, j}\right) .
$$

By the fixed point theorem of Brouwer, there exists $z_{0} \in \bar{D}(\lambda, r)$ such that $f\left(z_{0}\right)=z_{0}$. This implies $\operatorname{det}\left(\left\langle x_{i}, k_{j}\left(z_{0}\right)\right\rangle_{i, j}\right)=0$ and a contradiction is obtained. Thus $\Omega(\lambda) \cap \mathcal{C}_{n}(T)=$ $\emptyset$ and it follows that $\left((T-\lambda) y_{1}, y_{2}, \ldots, y_{n}\right) \notin \operatorname{cl}\left(\mathcal{C}_{n}(T)\right)$. The proof of $(i i)$ is complete.

Arguing inductively, we deduce the following corollary:

Corollary 5. Let $T$ be an n-multicyclic operator, $\left(x_{1}, x_{2}, \ldots, x_{n}\right) \in \mathcal{C}_{n}(T)$ and $P_{1}, P_{2}, \ldots, P_{n}$ be polynomials. Then

1. $\left(P_{1}(T) x_{1}, P_{2}(T) x_{2}, \ldots, P_{n}(T) x_{n}\right) \notin \mathcal{C}_{n}(T) \Leftrightarrow\left[\bigcup_{i=1}^{n} \mathcal{Z}\left(P_{i}\right)\right] \cap \mathcal{B}(T) \neq \emptyset$.

2. $\left(P_{1}(T) x_{1}, P_{2}(T) x_{2}, \ldots, P_{n}(T) x_{n}\right) \notin \operatorname{cl}\left(\mathcal{C}_{n}(T)\right) \Rightarrow\left[\bigcup_{i=1}^{n} \mathcal{Z}\left(P_{i}\right)\right] \cap \operatorname{int}(\mathcal{B}(T)) \neq \emptyset$.

3. $\left[\bigcup_{i=1}^{n} \mathcal{Z}\left(P_{i}\right)\right] \cap \mathcal{B}_{a}(T) \neq \emptyset \Rightarrow\left(P_{1}(T) x_{1}, P_{2}(T) x_{2}, \ldots, P_{n}(T) x_{n}\right) \notin c l\left(\mathcal{C}_{n}(T)\right)$.

Here $\mathcal{Z}(P)$ stands for the set of all zeros of the polynomial $P$.

Proof of Theorem 10. The implication $(i) \Rightarrow(i i)$ is direct from Proposition 6 .

$($ ii $) \Rightarrow(i)$. Let $X=\left(x_{1}, x_{2}, \ldots, x_{n}\right)$ be in the interior of $\mathcal{X}^{(n)} \backslash \mathcal{C}_{n}(T)$ and let $\left(y_{1}, y_{2}, \ldots, y_{n}\right)$ be a cyclic $n$-tuple of $T$. For $1 \leq i \leq n$, there exist polynomials $\left\{P_{i, 1}\right.$, $\left.P_{i, 2}, \ldots, P_{i, n}\right\}$ such that

$$
\left.\| \sum_{j} P_{i, j}(T) y_{i}-x_{i}\right) \|<\frac{\operatorname{dist}\left(X, \mathcal{C}_{n}(T)\right)}{n} .
$$


Then, it follows that

$$
\left\|\sum_{i=1}^{n}\left(\sum_{j} P_{i, j}(T) y_{i}-x_{i}\right)\right\|<\operatorname{dist}\left(X, \mathcal{C}_{n}(T)\right) .
$$

Using Smith's form of the polynomial matrix $\left(P_{i, j}(T)\right)_{i, j}$ (see [10], page 311), there exists a family of non-vanishing polynomials $\left(P_{1}, P_{2}, \ldots, P_{n}\right)$ and $\left(y_{1}^{\prime}, y_{2}^{\prime}, \ldots, y_{n}^{\prime}\right) \in \mathcal{C}_{n}(T)$ such that $\left(P_{1}(T) y_{1}^{\prime}, P_{2}(T) y_{2}^{\prime}, \ldots, P_{n}(T) y_{n}^{\prime}\right)$ is an interior point of $\mathcal{X}^{(n)} \backslash \mathcal{C}_{n}(T)$. In particular, $\left(P_{1}(T) y_{1}^{\prime}, P_{2}(T) y_{2}^{\prime}, \ldots, P_{n}(T) y_{n}^{\prime}\right) \notin \operatorname{cl}\left(\mathcal{C}_{n}(T)\right)$. By Corollary 5 , we have $\left[\bigcup_{i=1}^{n} \mathcal{Z}\left(P_{i}\right)\right] \cap$ $\operatorname{int}(\mathcal{B}(T)) \neq \emptyset$ and hence $\operatorname{int}(\mathcal{B}(T)) \neq \emptyset$. Now $\mathcal{B}_{a}(T) \subset \operatorname{int}(\mathcal{B}(T)) \subset \operatorname{cl}\left(\mathcal{B}_{a}(T)\right)$ implies that $\mathcal{B}_{a}(T) \neq \emptyset$.

Let $\omega=\left(\omega_{n}\right)_{n \geq 0}$ be nonnegative numbers. The Beurling space associated with $\omega$ is given by

$$
H_{\omega}^{2}=\left\{f ; f(z)=\sum_{n \geq 0} a_{n} z^{n} \text { and } \sum_{n \geq 0}\left|a_{n}\right|^{2} \omega_{n}^{2}<+\infty\right\} .
$$

The shift operator $M_{z, \omega}$ is the multiplication operator in $H_{\omega}^{2}$ by the independent variable $z$. The operator $M_{z, \omega}$ is bounded if and only if $\sup _{n} \omega_{n+1} / \omega_{n}<+\infty$. Moreover $M_{z, \omega}$ is cyclic with 1 as a cyclic vector. Set $r_{2}(\omega)=\liminf _{n} \omega_{n}^{1 / n}$. Then $B_{a}(T)=D\left(0, r_{2}(\omega)\right)$, (see [26], for example).

We have the following corollary:

COROllary 6. Under the notations above $C\left(M_{z, \omega}\right)$ is dense if and only if $r_{2}(\beta)=0$.

The preceding corollary includes the example given by D. A. Herrero for the shift operator on the usual Hardy space (see [12, Example 12]).

In [27] E. Thomson has proved that analytic bounded point evaluations exist for pure cyclic subnormal operators. Hence we have

Corollary 7. Let $T$ be a pure cyclic subnormal operator. Then $\operatorname{cl}(\mathcal{C}(T)) \neq \mathcal{X}$.

In [3] S. Ansari and P. Bourdon have shown that if $T^{n}$ is cyclic for every $n$, then the set of cyclic vectors of $T$ is dense in $\mathcal{X}$. T. Trent gave the same result for subnormal operators (see [29]).

Applying Theorem 10, the above result is extended to the class of all multicyclic operators.

TheOREM 11. Let $T \in \mathcal{L}(\mathcal{X})$ be an $n$-multicyclic operator defined on a Banach space $\mathcal{X}$. If $T^{m} \in \mathcal{C}_{n}(\mathcal{X})$, for every nonnegative number $m$, then $\mathcal{C}_{n}(T)$ is dense in $\mathcal{X}^{(n)}$.

Proof. It suffices to show that $\mathcal{B}_{a}(T)=\emptyset$. Seeking a contradiction, suppose $\mathcal{B}_{a}(T) \neq$ $\emptyset$. For $m \geq 2$, let $\left(x_{1}, x_{2}, \ldots, x_{n}\right)$ be a cyclic $n$-tuple of $T^{m}$. Since $T x_{1} \in \mathcal{X}$ there are polynomials $\left\{\left(P_{1, s}\right)_{s \geq 0},\left(P_{2, s}\right)_{s \geq 0}, \ldots,\left(P_{n, s}\right)_{s \geq 0}\right\}$ such that $\sum_{j=1}^{n} P_{j, s}\left(T^{m}\right) x_{j} \rightarrow T x_{1}$. Now since $\mathcal{C}\left(T^{m}\right) \subset \mathcal{C}(T)$ there exists an analytic function $k: \lambda \in \mathcal{B}_{a}(T) \mapsto k(\lambda) \in$ $N(T-\lambda)^{*}$ such that $\left\langle x_{j}, k(\lambda)\right\rangle=\delta_{1, j}$. Then for every $\lambda \in \mathcal{B}_{a}(T)$ we have

$$
\left|P_{1, s}\left(\lambda^{m}\right)-\lambda\right|=\left|\left\langle\sum_{j=1}^{n} P_{j, s}\left(T^{m}\right) x_{j}, k(\lambda)\right\rangle-\lambda\right|
$$




$$
\begin{aligned}
& =\left|\left\langle\sum_{j=1}^{n} P_{j, s}\left(T^{m}\right) x_{j}, k(\lambda)\right\rangle-\lambda\left\langle x_{1}, k(\lambda)\right\rangle\right| \\
& =\left|\left\langle\sum_{j=1}^{n} P_{j, s}\left(T^{m}\right) x_{j}-T x_{1}, k(\lambda)\right\rangle\right| \\
& \leq\left\|\sum_{j=1}^{n} P_{j, s}\left(T^{m}\right) x_{j}-T x_{1}\right\|\|k(\lambda)\| .
\end{aligned}
$$

Since the mapping $\lambda \mapsto\|k(\lambda)\|$ is bounded on compact subsets of $\mathcal{B}_{a}(T)$ we deduce that $P_{1, s}\left(z^{m}\right)$ is pointwise convergent in $\mathcal{B}_{a}(T)$ to the identity map $z \mapsto z$. Uniqueness of limits insures that $e^{\frac{2 i \pi}{m}} z \notin \mathcal{B}_{a}(T)$. Hence, if $T^{m} \in \mathcal{C}_{n}(\mathcal{X})$ for every $m \geq 2$, then for every nonzero $z \in \mathcal{B}_{a}(T)$, it will be that $e^{\frac{2 i \pi}{m}} z \notin \mathcal{B}_{a}(T)$. This provides a contradiction since $\mathcal{B}_{a}(T)$ is an open set.

REMARK 7 . One may try to relax in Theorem 11 the assumption $T^{m} \in \mathcal{C}_{n}(\mathcal{X})$, for every nonnegative number $m$ to the weaker one, $T^{m}$ is multicyclic for every nonnegative number $m$. The shift operator $S$ on the usual Hardy space $H^{2}$ is illuminating in this sense. Indeed, $S^{n}$ is $n$-multicyclic with $\left(1, z, \ldots, z^{n-1}\right)$ as cyclic $n$-tuple. On the other hand, clearly the set of analytic bounded evaluations has nonempty interior and thus $\mathcal{C}(T)$ is not dense.

Acknowledgements. The authors are indebted to the referee for many helpful suggestions.

\section{References}

[1] J. Akeroyd and E. G. Saleeby, A class of $\mathcal{P}^{t}(d \mu)$ spaces whose point evaluations vary with $t$, Proc. Amer. Math. Soc. 127 (1999), 537-542.

[2] E. Albrecht and J. Eschmeier, Analytic functional models and local spectral theory, Proc. London Math. Soc. 75 (1997), 323-348.

[3] S. I. Ansari and P. S. Bourdon, Some properties of cyclic operators, Acta Sci. Math. (Szeged) 63 (1997), 195-207.

[4] C. Apostol, L. A. Fialkow, D. A. Herrero and D. Voiculescu, Approximation of Hilbert Space Operators II, Research Notes in Mathematics 102, Pitman, 1984.

[5] A. Bourhim, C. E. Chidume and E. H. Zerouali, Bounded point evaluations for cyclic operators, Proc. Amer. Math. Soc. 130 (2002), 543-548.

[6] J. B. Conway, The Theory of Subnormal Operators, Mathematical Surveys and Monographs 36, American Mathematical Society, 1991.

[7] J. Eschmeier and B. Prunaru, Invariant subspaces and localizable spectrum, Integral Equations Operator Theory 42 (2002), 461-471.

[8] N. Feldman, Pure subnormal operators have cyclic adjoint, J. Funct. Anal. 162 (1999), 379-399.

[9] J. Finch, On the local spectrum and the adjoint, Pacific J. Math. 94 (1981), 297-302.

[10] I. T. Gokhberg, P. Lancaster and L. Rodman, Matrix Polynomials, Academic Press, New York, 1982.

[11] D. A. Herrero, On multicyclic operators, Integral Equations Operator Theory 1 (1978), 57-102. 
[12] D. A. Herrero, The Fredholm structure of an n-multicyclic operator, Indiana Univ. Math. J. 36 (1987), 549-566.

[13] D. A. Herrero and L. Rodman, The multicyclic n-tuples of an n-multicyclic operator, and analytic structures on its spectrum, Indiana Univ. Math. J. 34 (1985), 619-629.

[14] K. B. Laursen and M. M. Neumann, An Introduction to Local Spectral Theory, London Math. Soc. Monographs, 2000

[15] J. E. McCarthy, Reflexivity of subnormal operators, Pacific J. Math. 161 (1993), 359-370.

[16] J. E. McCarthy and L. M. Yan, Bounded point evaluations on the boundaries of L regions, Indiana Univ. Math. J. 43 (1994), 857-883.

[17] M. Mbekhta, Généralisation de la décomposition de Kato aux opérateurs paranormaux et spectraux, Glasgow J. Math. 29 (1987), 159-175.

[18] M. Mbekhta, Résolvante généralisée et théorie spectrale, J. Operator Theory 21 (1989), 69-105.

[19] M. Mbekhta et A. Ouahab, Opérateur s-régulier dans un espace de Banach et théorie spectrale, Acta Sci. Math. (Szeged) 59 (1994), 525-543.

[20] M. Mbekhta et A. Ouahab, Perturbation des opérateurs s-réguliers, in: Topics in Operator Theory, Operator Algebras and Applications (Timişoara, 1994), Rom. Acad., Bucharest, 1995, 239-249.

[21] M. Mbekhta et E. H. Zerouali, Points d'évaluation pour les opérateurs cycliques ayant la propriété de Bishop ( $\beta$ ), J. Funct. Anal. 206 (2004), 69-86.

[22] M. Mbekhta and E. H. Zerouali, On the interior of bounded point evaluations, in preparation.

[23] V. Müller, On the regular spectrum, J. Operator Theory 31 (1994), 363-380.

[24] V. Müller, Spectral Theory of Linear Operators and Spectral Systems in Banach Algebras, Operator Theory Advances and Applications 139, Birkhäuser, 2003.

[25] T. L. Miller, V. G. Miller, and M. M. Neumann, Analytic bounded point evaluations for cyclic operators on Banach spaces, Integral Equations Operator Theory, to appear.

[26] A. L. Shields, Weighted shift operators and analytic function theory, Mathematical Surveys 13, American Mathematical Society, 1974, 49-128.

[27] E. Thomson, Approximation in the mean by polynomials, Ann. Math. 133 (1991), 477-507.

[28] T. Trent, $H^{2}(\mu)$ spaces and bounded point evaluations, Pacific J. Math. 80 (1979), 279-292.

[29] T. Trent, A characterization of $\mathcal{P}^{2}(\mu) \neq L^{2}(\mu)$, J. Funct. Anal. 64 (1985), 163-177.

[30] T. Trent, Sufficient conditions for bounded point evaluations, Integral Equations Operator Theory 13 (1990), 593-606.

[31] T. Trent and J. L. Wang, $\mathcal{P}^{2}(\mu)$ and bounded point evaluations, Proc. Amer. Math. Soc. 91 (1984), 421-425.

[32] F.-H. Vasilescu, Analytic Functional Calculus and Spectral Decomposition, Editura Academiei, Bucharest, 1982.

[33] L. Williams, Bounded point evaluations and the local spectra of cyclic hyponormal operators, Dynamic Systems and Applications 3 (1994), 103-112. 